\title{
General Qualifier
}

National Cancer Institute

\section{Source}

National Cancer Institute. General Qualifier. NCI Thesaurus. Code C27993.

A term that helps define and render another concept unique. 